\title{
Optical measurement of high-temperature melt flow rate
}

\author{
Benjamin Bizjan, ${ }^{1,2,}{ }^{*}$ Brane ŠıroK, ${ }^{1}$ Jinpeng Chen ${ }^{3}$ \\ ${ }^{1}$ University of Ljubljana, Faculty of Mechanical Engineering, Aškerčeva 6, 1000 Ljubljana, Slovenia \\ ${ }^{2}$ Abelium d.o.o., Research and Development, Kajuhova 90, 1119 Ljubljana, Slovenia \\ ${ }^{3}$ Guangdong University of Petrochemical Technology, 139 Guandu Erlu, Maoming, Guangdong 525000, China \\ *Corresponding author: benjamin.bizjan@fs.uni-lj.si
}

Received XX Month XXXX; revised XX Month, XXXX; accepted XX Month XXXX; posted XX Month XXXX (Doc. ID XXXXX); published XX Month XXXX

\begin{abstract}
This paper presents an optical method and system for contactless measurement of mass flow rate of melts by digital cameras. Proposed method is based on reconstruction of melt stream geometry and flow velocity calculation by crosscorrelation, and is very cost effective due its modest hardware requirements. Using a laboratory test rig with a small inductive melting pot and reference mass flow rate measurement by weighing, the proposed method was demonstrated to have an excellent dynamic response ( $0.1 \mathrm{~s}$ order of magnitude) while producing deviations from the reference of about $5 \%$ in the steady-state flow regime. Similar results were obtained in an industrial stone wool production line for two repeated measurements. Our method was tested in a wide range of melt flow rates $(0.05-1.2 \mathrm{~kg} / \mathrm{s})$ and did not require very fast cameras (120 frames per second would be sufficient for most industrial applications).
\end{abstract}

OCIS codes: (040.1490) Cameras; (100.2000) Digital image processing; (110.4153) Motion estimation and optical flow; (120.7250) Velocimetry; (240.6648) Surface dynamics; (280.2490) Flow diagnostics.

http://dx.doi.org/10.1364/AO.99.099999

\section{INTRODUCTION}

Accurate measurement of the mass flow rate of melts is one of essential requirements for optimal regulation of industrial processes such as casting of metals and stone wool production. With that said, melt flow measurements at the melting furnace outlet have so far been largely avoided due to many operational challenges such as very high melt temperatures and irregular melt stream geometry. Instead, the melt flow rate has usually been measured indirectly either from mass flows of input raw materials, or by weighing of solidified products at later stages of the production line using conveyor scales [1]. Such indirect mass flow measurements may suffer from poor accuracy if a significant amount of melt is lost between the furnace outlet and the conveyor scale location. For instance, in the stone wool industry the fraction of melt, which is not successfully fiberized and transported to later manufacturing phases, may exceed $20 \%$ of the gross mass flow of melt from the furnace [2]. Moreover, the significant lag between the melt outlet from the furnace and the product weighing location (up to the minute order of magnitude) limits the dynamic response of the measurement method, thus reducing the effectiveness of regulation of furnace and spinning machine operating parameters such as melt impingement position, flow rate of liquid binder and rotational speed of spinning wheels.

To overcome the disadvantages of indirect melt flow measurements, the mass flow of melt should be measured immediately at the melting furnace outlet. Due to harsh operating conditions, only some nonintrusive, contactless methods are suitable for this purpose. In transparent liquids such as water, most accurate non-intrusive flow rate measurements are based on the cross-correlation principle [3] using laser beams [4], ultrasound [5] or tracer dilution [6]. Since most mineral and metallic melts are not transparent, the above-mentioned penetrating optical methods are unsuitable for application. In the past, melt flow measurement with radioactive isotope tracers was tested with limited success, but this method is no longer used due to safety and environmental concerns [2]. Recently, Dubovikova et al. [7] evaluated the possibility of measuring the flow rate of liquid metals by contactless electromagnetic Lorentz force technique, which generates artificial vortices within the melt and then measures their velocity. While this method is showing some promising results with metallic melts, it cannot be applied to non-conductive melts used in the casting of nonmetals and in production of stone wool. A more generally applicable method was developed and tested by Chen et al [8] [9], who used a pneumatic system for generating visible flow disturbances on a falling stream of mineral melt. Melt stream shape and velocity were both calculated from camera recordings of the stream. Velocimetry was performed by cross-correlation of consecutive disturbance propagation images, with resulting melt flow rates in a good agreement with reference method measurements.

Nevertheless, due to a relatively complex and expensive set-up of this method, we have developed a simplified mass flow measurement system that relies on spontaneously occurring melt impurities and disturbances as velocimetry tracers, and will be presented in this paper. Accuracy of proposed method has been assessed by reference measurements of the melt mass flow by weighing of the melt 
accumulated in a tundish. Besides, the paper also presents the performance of our melt flow measurement method in an actual industrial manufacturing process of stone wool.

\section{EXPERIMENTAL SET-UP AND METHODOLOGY}

\section{A. Experimental set-up}

Experiments for evaluation of proposed method for melt flow rate measurement were performed in a laboratory environment. In the first step, the inputmaterial (stone wool) was melted in an induction melting furnace (Fig. 1a) with approximately $20 \mathrm{~kW}$ power. Since stone wool is not electrically conductive, it was heated indirectly inside a graphite melting pot. The pot itself was heated by eddy currents generated by a surrounding water-cooled induction winding. Ceramic wool insulation was used to minimize heat losses and protect the induction winding from overheating.
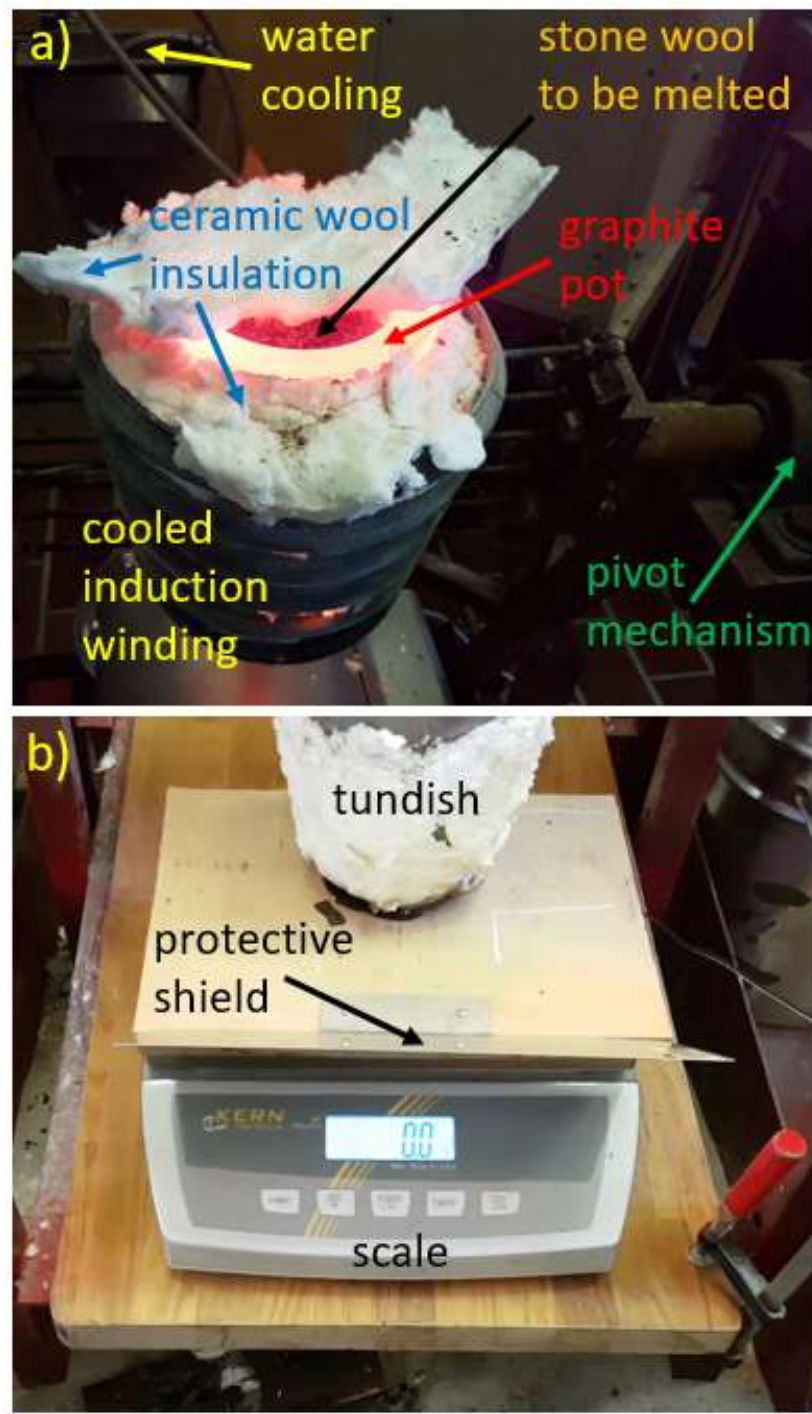

Fig. 1. Induction melting furnace with graphite pot (a); tundish on the scale (b).

When reaching the temperature of $1450^{\circ} \mathrm{C}$ (measured by a pyrometer), the melt was poured from the melting pot into a tundish placed on top of a lab scale KERN FKB 15K0.5A (Fig. 1b) with $\pm 0.5 \mathrm{~g}$ precision [10]. Pouring was carried out by rotating the pot using a pivot mechanism. A schematic view of experimental set-up is presented in Fig. 2.

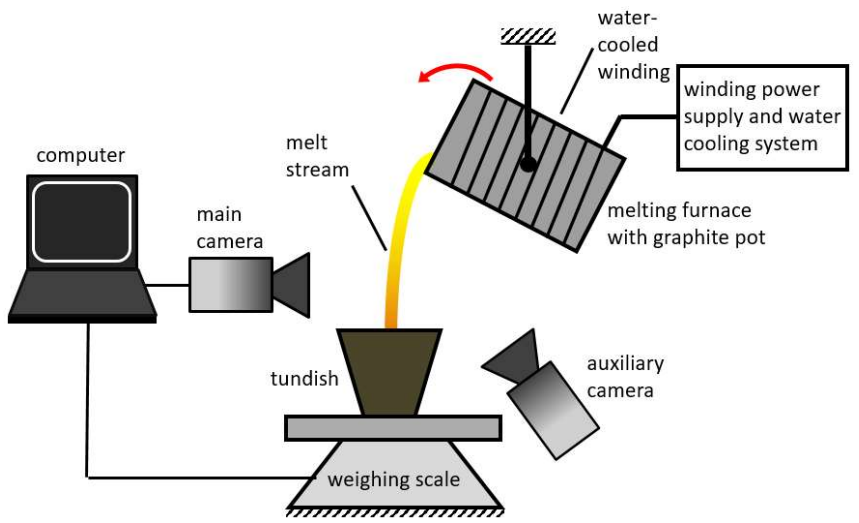

Fig. 2. Experimental set-up for measuring the mass flow rate of melt.

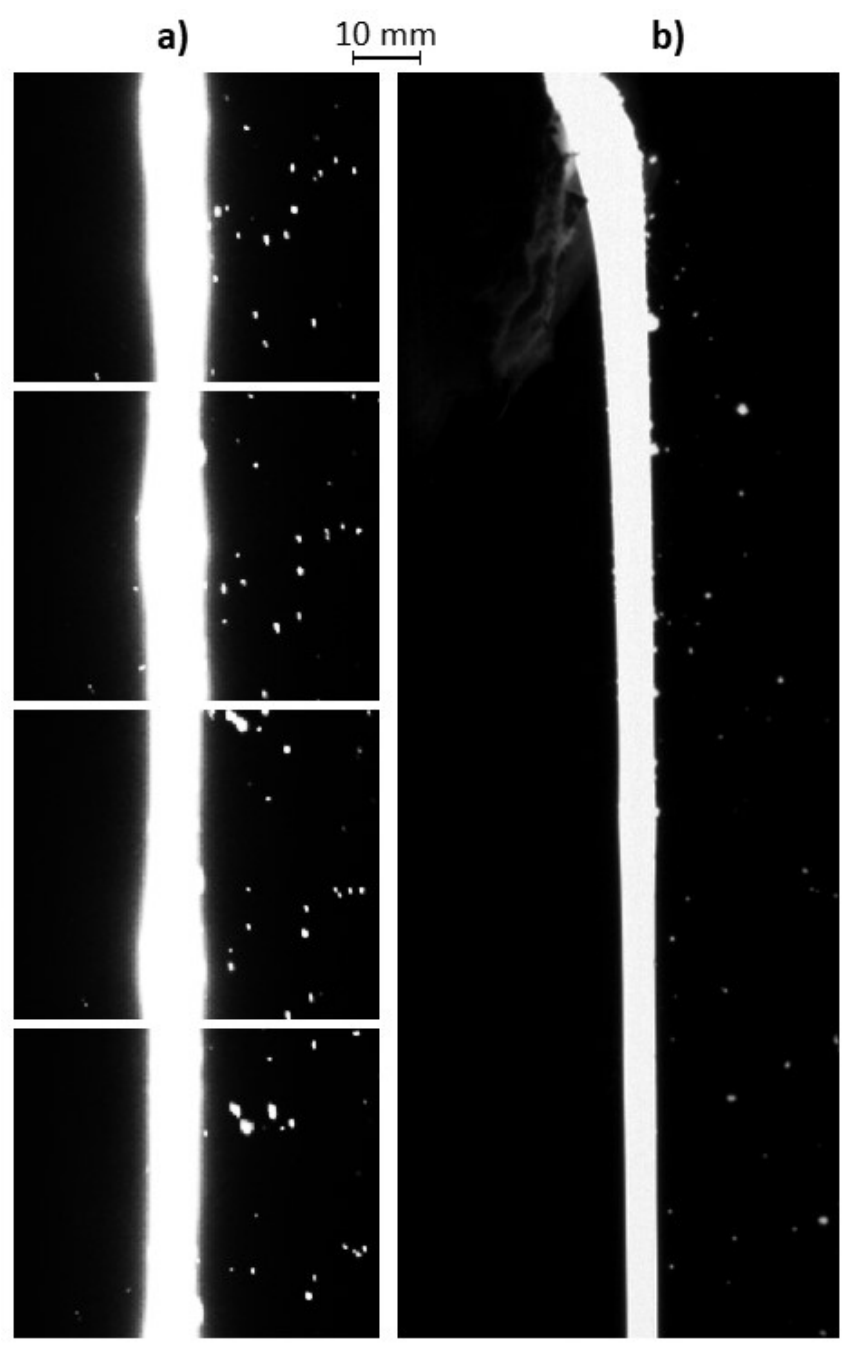

Fig. 3. Sample images: (a) main camera, $8 \mathrm{~ms}$ frame separation (frontal melt stream view) ; (b) auxiliary camera (side view of melting pot and melt stream).

The scale readout was acquired by a computer (10 readings per second) and synchronized with a camera (Basler acA 1300-30gm [11] with $8 \mathrm{~mm}$ lens) which captured successive images of the falling melt 
stream at 125 frames per second and $16 \mu$ s exposure time. Output images were 706 pixels wide and 172 pixels high. A free melt stream flowing from a furnace outlet or, in our case, an open pot, is known to have an elliptical rather than circular cross-section [8]. To estimate the eccentricity of the stream cross-section, an auxiliary camera was installed horizontally, but at a $90^{\circ}$ angle to the optical axis of the main camera so that melt flow was also visualized in a side view (instead of a second camera, a mirror can also be used).

Sample images from the main and the auxiliary camera are shown in Fig. 3a and 3b, respectively. Images from both cameras had the same pixel size, $0.27 \mathrm{~mm}$. Region of interest of the main camera corresponds to the lowermost viewing area of the auxiliary camera. Note the downwards-propagating flow disturbances in main camera frames (Fig. 3a), and contraction of the melt stream as melt is accelerated by the gravity (Fig. 3b).

After pouring the first batch of melt, new stone wool was added into the graphite pot, melted and heated to the same temperature. Then, the pouring process and the corresponding measurements were repeated. Due to changing melting pot angle and rotation speed, the mass flow rate of melt fluctuated randomly and quite significantly in both pouring experiments, but mostly stayed between $100 \ldots 200 \mathrm{~g} / \mathrm{s}$. This way, we were able to evaluate the dynamic response of our optical measurement method while still considering the second pouring experiment as a repetition of the first experiment.

\section{B. Methodology of mass flow measurement}

In the first step of experimental data postprocessing, the reference mass flow of melt was determined from scale weight readouts (Fig. 4). In Fig. 4 , the time $t=0$ marks the moment when melt first reached the tundish. Note that initially $m=0$ as the scale was zeroed prior to melt pouring to disregard the weight of the empty tundish. From Fig. 4 it is evident that the mass of melt in the tundish rose mostrapidly in the first 6 seconds of measurement. After $t=6 \mathrm{~s}$, the mass of melt increased more slowly until reaching its final value just under $1 \mathrm{~kg}$ between $t=7 \mathrm{~s}$ and $t$ $=9 \mathrm{~s}$, when the melting pot was completely drained. The flow of melt became intermittent (i.e. dripping) after $t \approx 6.5 \mathrm{~s}$, which is why we have decided to limit the time interval for analysis of recorded data to $t=0 \ldots 6$ s. Selected time interval resembles a typical operating regime of melting furnaces in stone wool production lines.

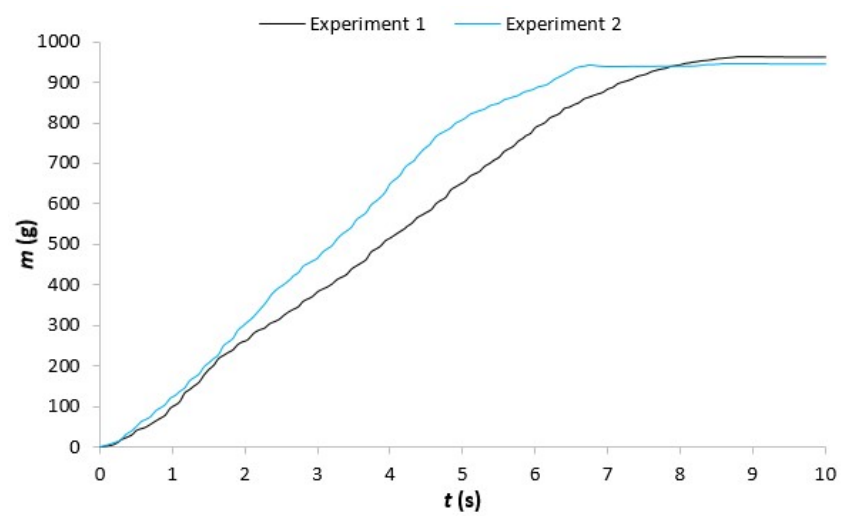

Fig. 4. Melt mass increase detected by the scale.

The reference mass flow of melt $Q_{\mathrm{mr}}$ was calculated by Eq. (1) as a numerical derivative of mass readings. In Eq. (1), i is the index of the current scale reading, and $\Delta t=0.1 \mathrm{~s}$ is the sampling time of acquired signal.

$$
Q_{m r}(t)=\frac{d m(t)}{d t} \approx \frac{m\left(\mathrm{t}_{i+1}-t_{i-1}\right)}{2 \Delta t} .
$$

In the next step, the mass flow of melt was calculated optically from recorded imaging data. The basic expression for mass flow rate calculation is given by Eq. (2), which is a simplified form of the mass conservation equation. In Eq. (2), $\rho$ denotes melt density and $v$ average melt velocity across the melt cross-section area $A$ normal to the flow direction (a scheme is provided in Fig. 5). Considering melt temperature $\left(1450^{\circ} \mathrm{C}\right)$ and chemical composition $\left(40 \% \mathrm{Si}_{2}, 25 \% \mathrm{CaO}, 6 \% \mathrm{MgO}\right.$, $14 \% \mathrm{Al}_{2} \mathrm{O}_{3}, 9 \% \mathrm{Fe} 2 \mathrm{O} 3,3 \% \mathrm{Na}_{2} \mathrm{O}, 2 \% \mathrm{TiO}_{2}, 1 \% \mathrm{~K}_{2} \mathrm{O}$ ), the density $\rho=$ $2620 \mathrm{~kg} / \mathrm{m}^{3}$ was calculated according to Pye et al. [12].

$$
Q_{m}=\rho \cdot v \cdot A \text {. }
$$

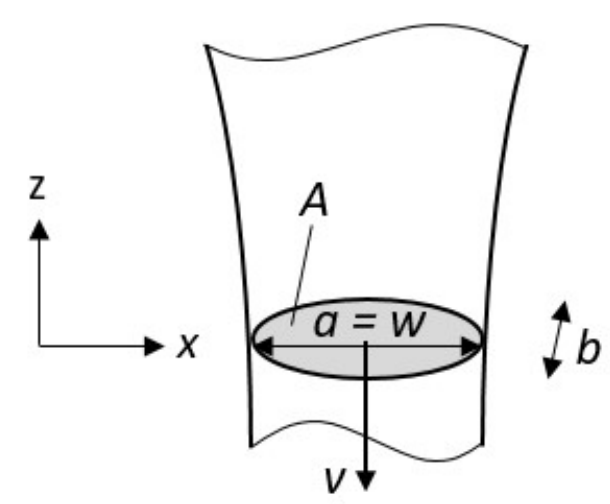

Fig. 5. Section of an elliptical melt stream with coordinate system.

The area $A=\pi a b / 4$ was calculated assuming an elliptical crosssection of the melt stream with major axis $a$ and minor axis $b$. Dimensions $a$ and $b$ were measured from main and auxiliary camera images, respectively. For both experiments, the ratio $b / a$ was determined to be the same and nearly constant in time, with a mean value of $b / a=0.63$. Considering this relation and introducing the melt stream width $w=a$, Eq. (2) can be rewritten as:

$$
Q_{m}=\rho \cdot v \cdot \frac{\pi}{4} \cdot \frac{b}{a} \cdot w^{2}=0.50 \rho \cdot v \cdot w^{2} .
$$

The width of the melt stream was obtained from main camera images as the distance between the left edge position $\left(x_{\mathrm{L}}\right)$ and the right edge position $\left(x_{\mathrm{R}}\right)$ of melt in images, $w=x_{\mathrm{R}}-x_{\mathrm{L}}$. In order to detect edge positions, images were first converted from 8-bit grayscale format (Fig. 6a) to 1-bit binary format (Fig. 6b). Then, an algorithm was used to clear binary images (Fig. 6c) of small white spots unconnected to the melt stream, which were caused by free falling shots and sparks.

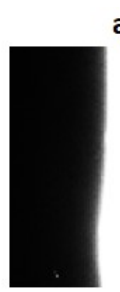

a)

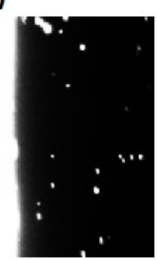

b)

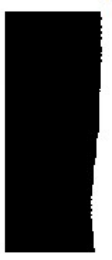

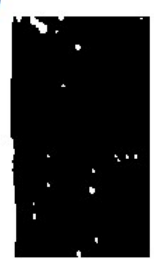

c)

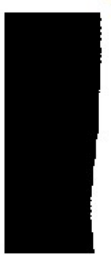

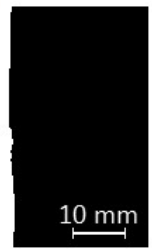

Fig. 6. Processing of melt stream images: (a) raw grayscale image; (b) binary image; (c) filtered binary image without small white spots.

Once a clear binary image has been obtained, positions of melt edges were detected by computing a profile of gray level gradient $G$ in a horizontal direction (Fig. 7) at each vertical position $z$ (unit: pixels) within the image of height $H=172$ pixels $(46.4 \mathrm{~mm})$. The gradient approach was proposed and presented in detail by Trdič et al. [13] who developed a system for measurement and control of the melt stream 
centerline position and angle. In contrast with the methodology presented in [13], no image downsampling was used, as it would significantly reduce the accuracy of melt width measurement.
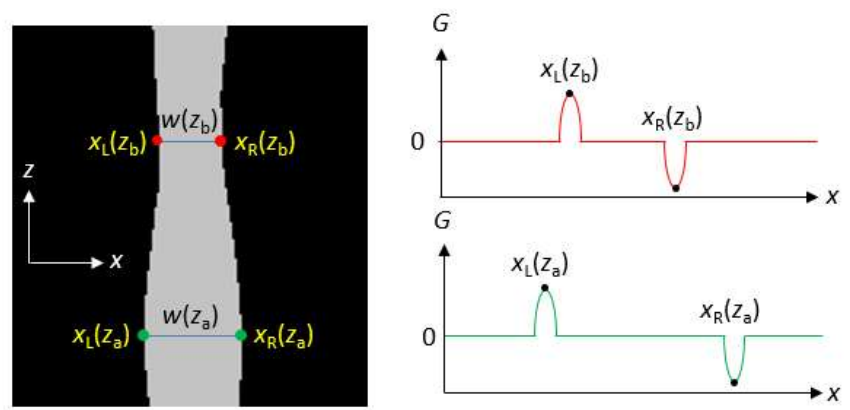

Fig. 7. Calculation of melt edges and width from profiles of gray level gradient.

In Fig. 7, a sample binary image of the melt stream is shown along with two selected vertical positions for melt edge position and width calculation. The left edge position $x \mathrm{~L}$ was obtained as the first gradient profile maximum, and the right edge position $x_{\mathrm{R}}$ as the last minimum of the gradient profile. For each of recorded images, a series of $x_{\mathrm{L}}$ and $x_{\mathrm{R}}$ positions was obtained. To reduce the effect of flow disturbances at melt edges on melt width calculation, an additional criterion was introduced: if $x_{\mathrm{L}}(\mathrm{z})-x_{\mathrm{L}}(z-1$ pixel $)>1$ pixel, then the particular value of $x_{\mathrm{L}}$ was disregarded and substituted by interpolation over remaining values (the same procedure was used for $x_{\mathrm{R}}$ ). In other words, if two neighboring points on the melt stream edge were more than one pixel apart in the horizontal direction, their value was not taken into consideration. Finally, the melt width $\mathrm{w}$ inside the calculation window can be calculated as:

$$
w=\frac{1}{H} \sum_{z=1}^{H}\left(x_{\mathrm{R}}(z)-x_{\mathrm{L}}(z)\right) .
$$

The last quantity to be determined is the average melt velocity $v$. In this study, velocity of the melt flow has been obtained by observing visible melt surface perturbations in consecutive images. Before discussing the velocimetry algorithm used, it is important to provide some remarks about correct camera settings. In some cases, including the laboratory experiment in this paper, there are many flow disturbances manifesting as local fluctuations in melt stream diameter (Fig. 3). Such disturbances may result from non-uniform temperature (and consequently, viscosity) distribution within the batch of melt, especially when melting time and melt volume are insufficient for optimal homogenization of melt, as was the case in this study. Nonuniform temperature of the melt surface can cause large fluctuations of the melt gray level [14], which may be useful for velocity calculation, but challenging for accurate melt width measurements (consider Fig. 8a for an example). Nevertheless, by setting the camera shutter time just high enough to achieve image saturation (i.e. white color) in the melt region, we were able to detect melt width reliably while retaining sufficient amount of visible flow disturbances for velocity calculation.

With that said, the situation in industrial melting processes is slightly different as the melt stream is typically more stable and with a rather smooth surface shape that is only occasionally disrupted by bulges and shots. Consider Fig. 8b for a sample image of a good quality industrial melt flow, and Results and discussions section for demonstration of melt flow rate measurements in an industrial stone wool production process. To compensate for the lack of large-scale perturbations that could be used for velocity calculation, camera shutter time can be reduced (or lens aperture closed) to observe smaller-scale structural inhomogeneities on the otherwise apparently smooth melt surface. a)

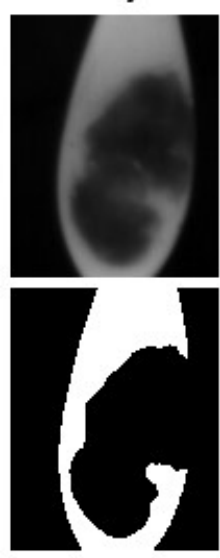

b)

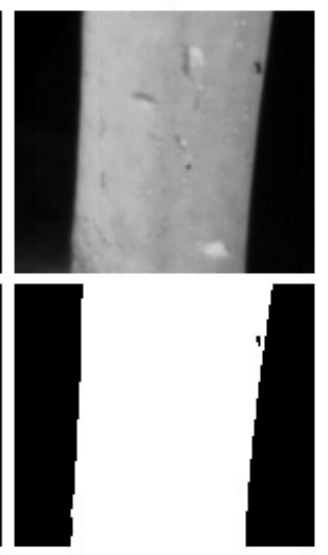

Fig. 8. Examples of poor quality (a) and good quality (b) melt flow. The upper and the lower row show grayscale images and converted binary images, respectively. Note the incorrect melt region detection in (a) due to colder and not fully melted material in the stream.

When melt visualization is performed correctly, velocity can be calculated using a relatively straightforward formulation. For presented experiments, let $\Delta t_{c}=0.008 \mathrm{~s}$ be the time separation between consecutive frames of the main camera, and $L$ the displacement of a disturbance in time $\Delta t_{\text {c }}$. Then, melt velocity can be computed as $v=L / \Delta t_{\text {c }}$ As shown by Gerogiorgis et al [15] who performed a CFD simulation of a falling melt stream, melt velocity is practically constant across the entire cross-section of the stream, except immediately downstream of the furnace outlet location. Consequently, the average melt velocity $v$ across the cross-section $A$ can be taken to be the same as velocity optically measured on the surface of the melt stream, without any correction necessary. Although the study presented in [15] was conducted for a circular melt stream, an elliptical cross section should produce very similar results, even at high eccentricities when flow resembles a falling liquid sheet [16].

As shown in Fig. 3, the framerate of recorded image sequences is large enough to observe the propagation of flow disturbances and other visible features in at least two consecutive images. Consequently, the disturbance displacement $L$ can be calculated by cross-correlation, following a methodology similar to the Particle image velocimetry (PIV) method [17]. Chen et al. [8] used cross correlation of spatial series of melt width ( $w$ as a function of $z$ ) for two consecutive images. Such simple correlation was possible because the diameter of the artificially generated flow disturbance was much higher than the mean melt width. In the present study however, spontaneous temporal variations of melt width have proven too infrequent for velocimetry by 1D crosscorrelation, though clearly visible. Instead, 2D correlation of grayscale images (Eq. (5)) has been employed to utilize all visible structures that move with the flow.

In order to minimize the computational expense of velocity calculation algorithm and thus the whole flow measurement method, we have not computed the full 2D cross-correlation function of consecutive image pairs. Instead, the following methodology has been developed:

1. Verify that in recorded images the melt stream flows vertically. This way, correlation must only be computed along the vertical axis $z$.

2. Select a subregion (correlation window) of the first image as gray level matrix $S_{1}$. In this study, the subregion size (width $\mathrm{x}$ height) was $228 \times 100$ pixels, starting at the image top position (Fig. 9). 
Image 1
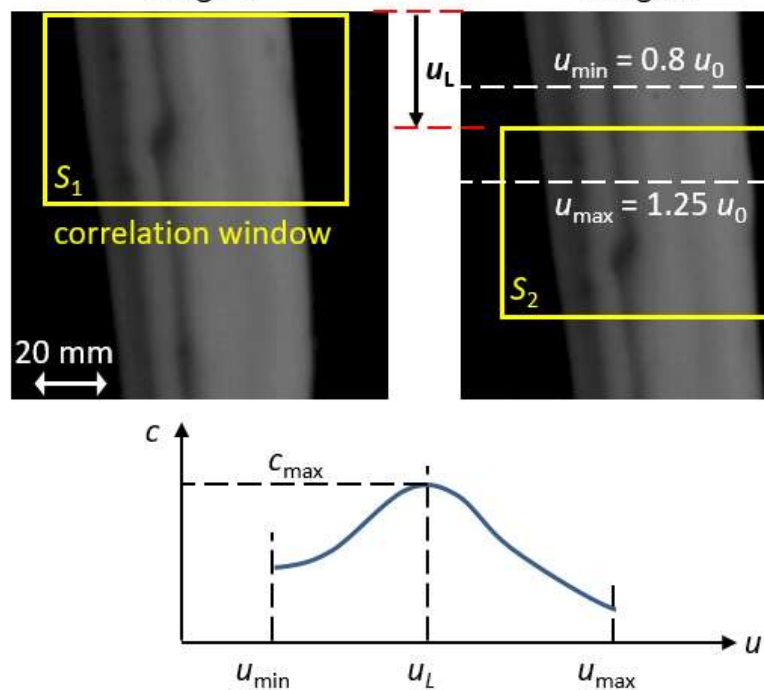

Image 2

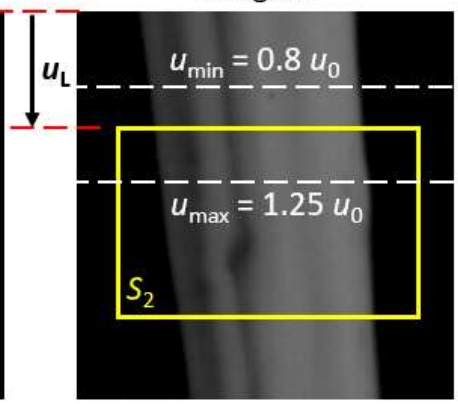

Fig. 9. Calculation of flow disturbance displacement $L$ by crosscorrelation.

3. Estimate velocity of the melt flow. Velocity estimation can be performed manually by taking a few random examples of feature movement between consecutive melt stream images, and measuring the movement in pixels $u$ (equal to optical velocity with unit pixel/frame). In our case, the estimate was $u_{0} \approx 54$ pixels/frame. Alternately, an initial estimate for melt velocity can be obtained from flow equations for falling melt jets ([18][19][20]), considering the current operating parameters of the melting furnace.

4. For a given set-up of a melting furnace, it is reasonable to expect that the actual value of $u$ will not change by more than $\pm 25 \%$, which is an empirical measure from plant operation experience with added safety margin. Therefore, $0.8 u_{0} \leq u \leq 1.25 u_{0}$, which in our case means 44 pixels $\leq u \leq 67$ pixels.

5. For the range of $u$ defined in the previous step (in integer increments), take a subregion of the second image, $S_{2}$ (same size as $S_{1}$ and one frame after the first image), so that the region of $S_{2}$ in the second image is located u pixels lower than $S_{1}$ in the first image (consider scheme in Fig. 9).

6. For all subregions $S_{2}$, compute 2D correlation of $S_{1}$ and $S_{2}$ by the following equation:

$$
c_{i}=\frac{\sum_{m} \sum_{n}\left(S_{1}(m, n)-M S_{1}\right)\left(S_{2}(m, n)-M S_{2}\right)}{\sqrt{\left(\sum_{m} \sum_{n}\left(S_{1}(m, n)-M S_{1}\right)^{2}\right)\left(\sum_{m} \sum_{n}\left(S_{2}(m, n)-M S_{2}\right)^{2}\right)}} .
$$

In Eq. (5), $0 \leq c_{\mathrm{i}} \leq 1$ is the correlation coefficient for $\mathrm{i}$-th element from the interval $0.8 u_{0} \leq \mathrm{u} \leq 1.25 u_{0}$. Indices $\mathrm{m}$ and $\mathrm{n}$ denote vertical and horizontal position in image subregions (in pixels). Parameters $\mathrm{MS}_{1}$ and $M S_{2}$ represent the mean value of the gray level of matrices $S_{1}$ and $S_{2}$, respectively. Definition of $c_{\mathrm{i}}$ was adopted from MATLAB function corr2 [21], which was used for calculations of correlation coefficients.

7. Let $c$ be the series of values of $c_{i}$. Find the maximum value of $c, c_{\max }$ and the corresponding value of $u, \mathrm{u}_{\mathrm{L}}$. Multiply $u_{\mathrm{L}}$ by pixel size to obtain $L$ in $[\mathrm{m}]$, then compute velocity $v$ in $[\mathrm{m} / \mathrm{s}]$.

8. Keep only velocity values where exactly one local maximum (i.e. peak) of $c$ is detected, and where the absolute difference between the maximum and minimum of $c$ is above a selected threshold, say 0.05 . Adjust this threshold if required to keep at least one velocity value per each interval of $0.1 \ldots 1 \mathrm{~s}$ length (depending on the application of the melt flow measurement method). Interpolate over excluded values if equidistant time series is required.

Now, the mass flow rate of melt can be calculated. It is worth noting that in most industrial processes, one $Q_{\mathrm{m}}$ reading per every few seconds is sufficient. The main reason for monitoring the melt stream with more than 100 frames per second is the velocity calculation algorithm due to its requirement that consecutive images must be taken shortly apart to capture every visible flow disturbance at least two times, thus maximizing the number of well-correlated image pairs. Once calculated, the series of $Q_{\mathrm{m}}$ can be downsampled as desired.

\section{RESULTS AND DISCUSSIONS}

\section{A. Laboratory experiment}

In this section, characteristic parameters for melt flow measurement will be presented as discussed in Methodology section. First, performance of the edge detection algorithm will be evaluated by qualitative inspection. In Fig. 10, 4 different examples of melt stream images recorded in both of our experiments are provided with 2 consecutive images for each example. Calculated positions of melt stream edges and centerlines are superimposed on raw images as red lines (gray in printed manuscript). From Fig. 10 it can be seen that melt edges are accurately detected even if large-amplitude disturbances occur in the flow (Fig. 10b and 9d). In addition, flying shots and sparks seen as small white spots in Fig. 10 do not interfere with the edge detection algorithm.

a)
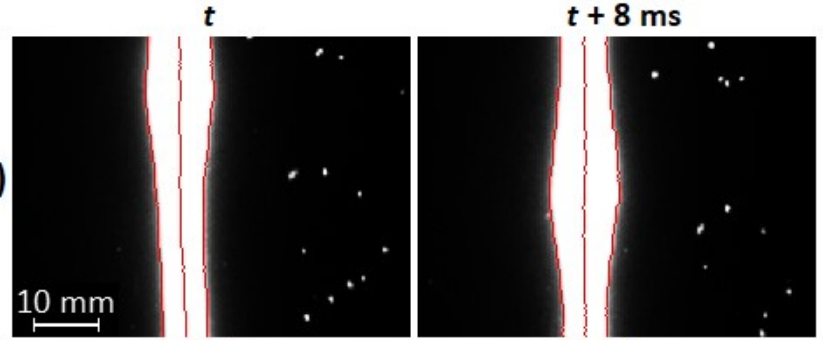

b)
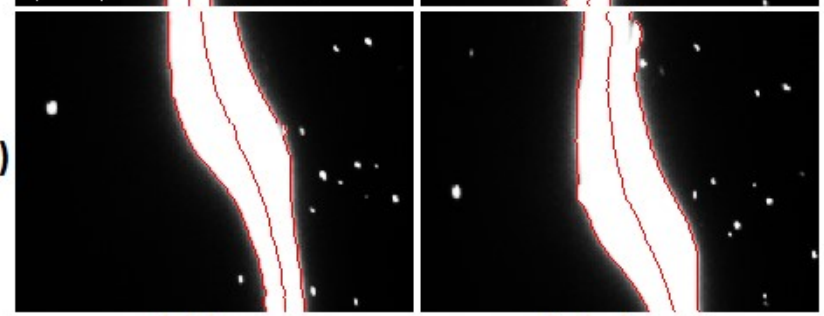

c)
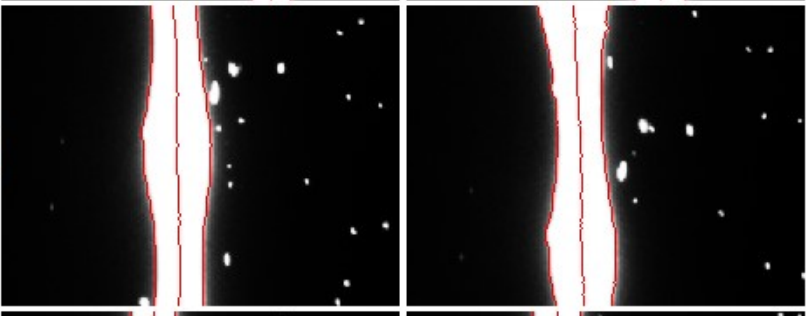

d)
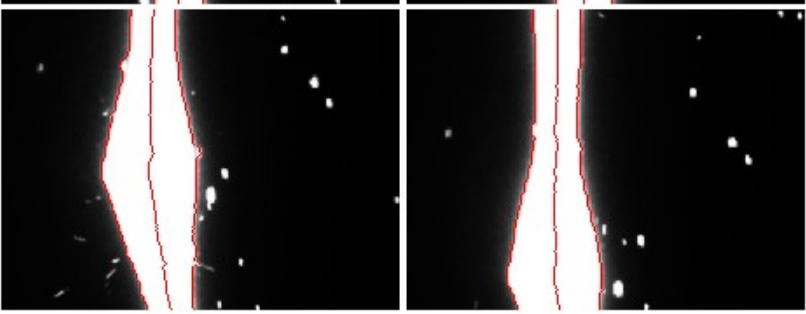

Fig. 10. Examples of melt edge and centerline detection. 
Note that centerlines drawn in Fig. 10 were obtained as mean positions between the left and the right melt edge. The mean centerline position of the melt stream $x_{c}$ will be defined by Eqs. (6) and (7):

$$
\begin{aligned}
& x_{c 0}=\frac{1}{2}\left(\mathrm{x}_{L}+\mathrm{x}_{R}\right) . \\
& \boldsymbol{x}_{\boldsymbol{c}}=\boldsymbol{x}_{\boldsymbol{c} \mathbf{0}}-\overline{\boldsymbol{x}_{\boldsymbol{c} \mathbf{0}}} .
\end{aligned}
$$

Time series of melt stream width and centerline position are shown in Fig. 11. In the time interval $0<t<1 \mathrm{~s}$, melt width can be seen to increase to about $9 \mathrm{~mm}$, while melt stream position experiences large fluctuations. Later, both $w$ and $x_{c}$ tend to stabilize, though still oscillating. Such behavior can be explained by the fact that in the time interval between $0 \mathrm{~s}$ and $1.5 \mathrm{~s}$, flow transition occurs as melt flow rate reaches its nominal value (also consider Figs. 13 and 14 for mass flow rate diagrams). Once the flow has stabilized, fluctuations of $w$ and $x_{c}$ decrease in amplitude, and flow conditions resemble those in industrial melt pouring processes. Occasional spikes in melt stream position (at $t=5.2$ $\mathrm{s}$ for experiment 1 and $t=2.9 \mathrm{~s}$ for experiment 2 ) were caused by larger lumps of incompletely melted material that temporarily disturbed the melt flow. After $t=4.5 \mathrm{~s}$, melt width calculated from experiment 2 starts to drop, which can be linked to a reduction in melt flow rate when the melting pot was almost completely drained.

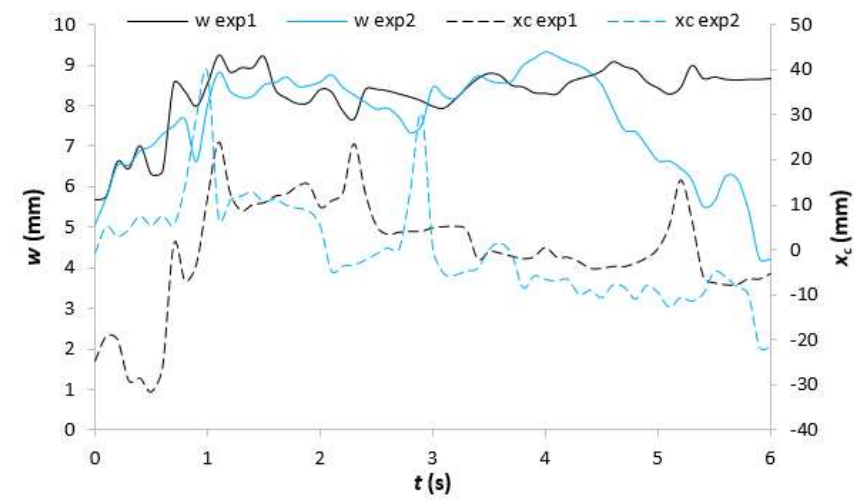

Fig. 11. Time series of melt stream width and centerline position for experiments 1 and 2 .

Unlike $w$ and $x_{c}$, the highest melt velocity $v$ (Fig. 12) was observed around $t=0 \mathrm{~s}$ and then slowly decreased with time. The decrease in velocity can be explained by a gradual reduction in height of the melting pot outflow above the viewing area as the pot was rotated from vertical to horizontal position (Fig. 2). Also noticeable are slow oscillations of velocity (particularly for experiment 1 ), which were likely caused by melt splashing in the pot and hydrodynamic instability of the jet [19].

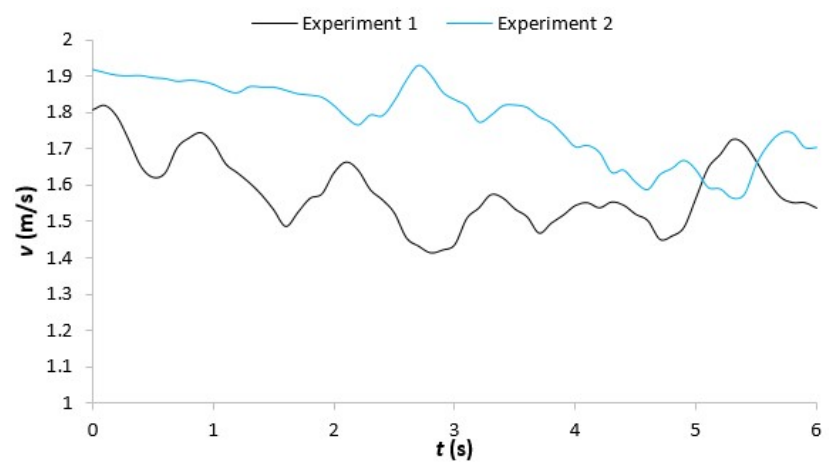

Fig. 12. Time series of melt stream velocity for experiments 1 and 2.
At this point, we can evaluate the accuracy of presented method for measurement of melt mass flow rate. For this purpose, both calculated and reference mass flows ( $Q_{\mathrm{m}}$ and $Q_{\mathrm{mr}}$, respectively) are plotted in Figs. 13 and 14. Also, measurement error will be analyzed by providing rootmean-square (RMS) values of relative errors in $1 \mathrm{~s}$ long intervals $\left(E_{\mathrm{RMS}}\right.$, Eq. (8)). Besides $E_{\mathrm{RMS}}, E_{\mathrm{A}}$ will be defined as the arithmetic mean of relative errors.

$$
E_{R M S}(t)=\sqrt{\frac{\Delta t}{Q_{m r}} \sum_{t-0.5 s}^{t+0.5 s}\left(Q_{m}-Q_{m r}\right)^{2}} .
$$

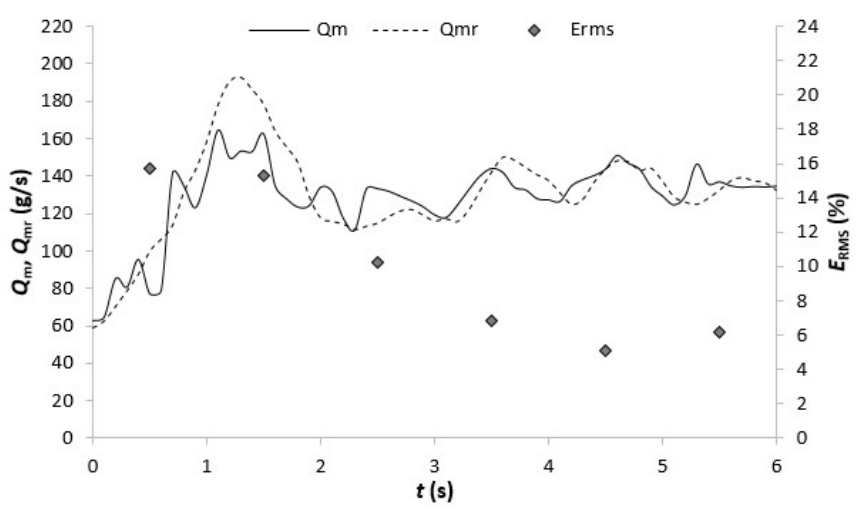

Fig. 13. Measured and reference mass flow rate of melt with RMS error value (experiment 1 ).

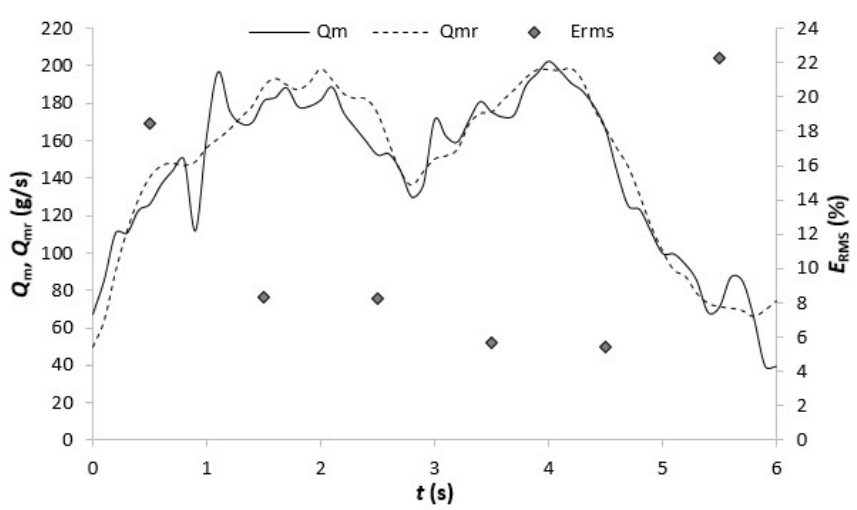

Fig. 14. Measured and reference mass flow rate of melt with RMS error value (experiment 2).

As can be seen in Figs. 13 and 14, there is a good agreement between reference and optically measured melt flow rates. The time series of $Q_{\mathrm{m}}$ follows the reference $Q_{\mathrm{mr}}$ series with a low shift (mostly under $0.1 \mathrm{~s}$ ), indicating a good dynamic response. Largest measurement errors have occurred in transitional flow regimes at the beginning and the end of measurements, with $E_{\mathrm{RMS}} \approx 15 \%$ for experiment $1(t<2 \mathrm{~s})$ and $E_{\mathrm{RMS}} \approx$ $20 \%$ for experiment $2(t<1 \mathrm{~s}$ and $t>5 \mathrm{~s}$ ). Once the melt flow has stabilized, $E_{\mathrm{RMS}}$ is observed to drop to below $10 \%$, reaching a minimum of about $5 \%$ for both experiments.

To expand the measurement error analysis, values of $Q_{\mathrm{m}}$ from both experiments were joined and binned into 7 intervals from $60 \mathrm{~g} / \mathrm{s}$ to 200 $\mathrm{g} / \mathrm{s}$ (Table 1). Mean and RMS values of error ( $E_{\mathrm{A}}$ and $E_{\mathrm{RMS}}$, respectively) were calculated, with $E_{\mathrm{RMS}}$ now defined as RMS value of each particular data bin. We assess $E_{\mathrm{RMS}}$ to be a more realistic error measure than $E_{\mathrm{A}}$, as the positive and negative values of $E_{\mathrm{A}}$ can cancel out, producing almost zero values (consider bins 100-120 g/s and 180-200 g/s). On the other 
hand, $E_{\mathrm{A}}$ provides information whether $Q_{\mathrm{mr}}$ is overestimated (positive sign), or underestimated (negative sign).

Table 1. Measurement errors as a function of the mass flow magnitude.

\begin{tabular}{cccc}
\hline $\begin{array}{c}Q_{\mathrm{m}} \\
(\mathrm{g} / \mathrm{s})\end{array}$ & $\begin{array}{c}\text { fraction } \\
(\%)\end{array}$ & $\begin{array}{c}E_{\mathrm{A}} \\
(\%)\end{array}$ & $\begin{array}{c}E_{\mathrm{RMS}} \\
(\%)\end{array}$ \\
\hline $60-80$ & 6.72 & -1.25 & 17.88 \\
$80-100$ & 8.40 & 13.87 & 17.45 \\
$100-120$ & 7.56 & 0.01 & 12.04 \\
$120-140$ & 34.45 & -1.26 & 9.19 \\
$140-160$ & 18.49 & -2.61 & 11.08 \\
$160-180$ & 15.13 & -1.48 & 6.29 \\
$180-200$ & 10.08 & -0.32 & 7.42 \\
\hline
\end{tabular}

Table 1 suggests that $Q_{\mathrm{mr}}$ was significantly overestimated in the range $80 \mathrm{~g} / \mathrm{s}<Q_{\mathrm{m}}<120 \mathrm{~g} / \mathrm{s}$, while in other value ranges, it was around 0 or slightly underestimated. Speaking of RMS errors, largest $E_{\mathrm{RMS}}$ values were computed for $Q_{\mathrm{m}}<100 \mathrm{~g} / \mathrm{s}$. As said, these relatively low mass flow rates occurred during flow transitions, when the value of $Q_{\mathrm{m}}$ was changing rapidly, and would not yield such high $E_{\mathrm{RMS}}$ values under steady flow conditions. In measurement range $120 \mathrm{~g} / \mathrm{s}<Q_{\mathrm{m}}<200 \mathrm{~g} / \mathrm{s}$, which make for $78.2 \%$ of all measured values, ERMS was mostly below $10 \%$.

\section{Uncertainty of measurement}

Reported measurement errors are closely related to the uncertainty of melt flow measurement. Considering the fact that flow rate is measured optically, the main effect contributing to higher measurement uncertainty at lower flow rates is the finite size of pixels in melt stream images. At $Q_{\mathrm{m}}=200 \mathrm{~g} / \mathrm{s}$, melt width w was about $9 \mathrm{~mm}$ (33 pixels), while at $Q_{\mathrm{m}}=50 \mathrm{~g} / \mathrm{s}$, it was reduced to $5 \mathrm{~mm}$ (18.5 pixels). The maximum $w$ calculation error due to pixel size is $0.5 / 33=1.5 \%$ at $200 \mathrm{~g} / \mathrm{s}$ and $2.7 \%$. Since $w$ is computed by averaging over multiple image lines, these errors may partly cancel out, though. Note that pixel size also contributes to measurement uncertainty in obtaining the displacement $L$, which is used to the velocity calculation in the same way through measuring - a maximum of $1.8 \%$ calculation error for $u=54$ pixels/frame. Considering the fact that $Q_{m} \propto v w^{2}$, maximum $Q_{\mathrm{m}}$ calculation errors due to finite pixel size can be estimated to $5.4 \%$ and $9.9 \%$, respectively. To reduce this source of measurement uncertainty, there are two possible solutions. Image resolution can be increased to accommodate at least 100 pixels across the width of the melt stream and $u=100$ pixels/frame, which would reduce the maximum $Q_{\mathrm{m}}$ error due to pixel size to $1.5 \%$. Alternately, a subpixel interpolation scheme could be employed, which would require a different, more computationally expensive image processing algorithm.

\section{B. Industrial environment measurements}

Besides laboratory experiments, imaging data from an industrial stone wool production process will also be used to demonstrate the performance of presented method for measurement of the mass flow rate of melt. Melt flow rate was measured above the impingement point of the melt stream on the first wheel of a spinning machine (Fig. 15). Two image sequences were recorded 2 minutes apart using the same imaging setup. Operating parameters of production line were kept constant during measurements.

For this experiment, camera shutter time and aperture settings were adjusted to capture structural inhomogeneities such as solid shots at the melt jet surface, which are clearly visible as moving darker spots and have served as main features for velocity calculation. In recorded images, larger flow disturbances are occasionally observed as local anomalies at melt stream edges (Fig. 15, bottom left corner of the calculation window). Note that prior to flow rate calculation, images were rotated so that melt propagation in calculation windows was in a vertical direction.

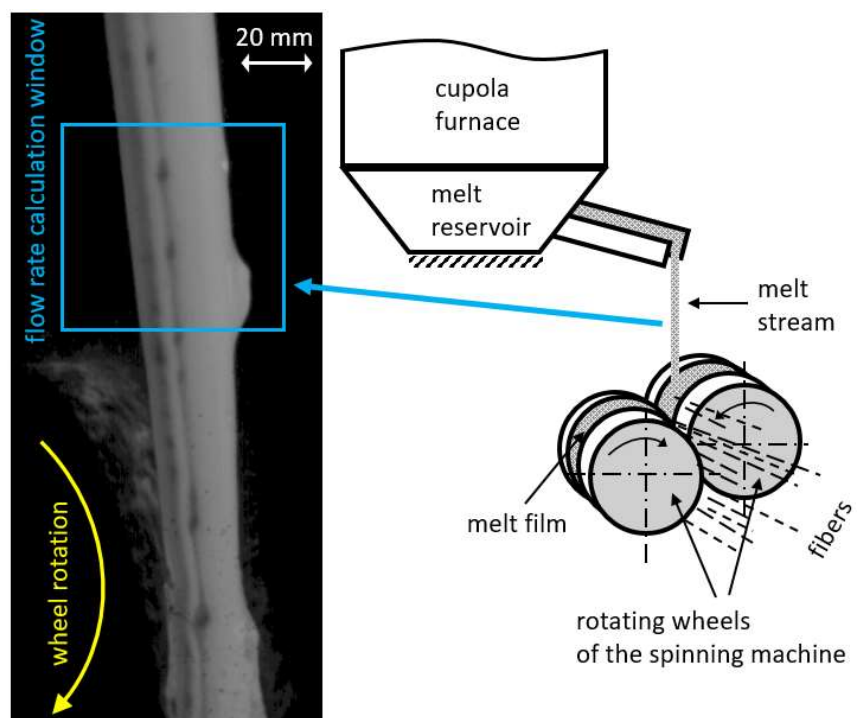

Fig. 15. Chosen positions for melt flow rate measurements in a stone wool production line, with sample camera images recorded at these positions.

In Fig. 16, calculated mass flow rate of melt is shown for both measurements (M1 and M2 in the graph legend) along with the centerline position of melt $x_{c}$. Due to design of the melt feeding system, the melt stream cross-section had a relatively high eccentricity $(b / a \approx$ $0.30)$.

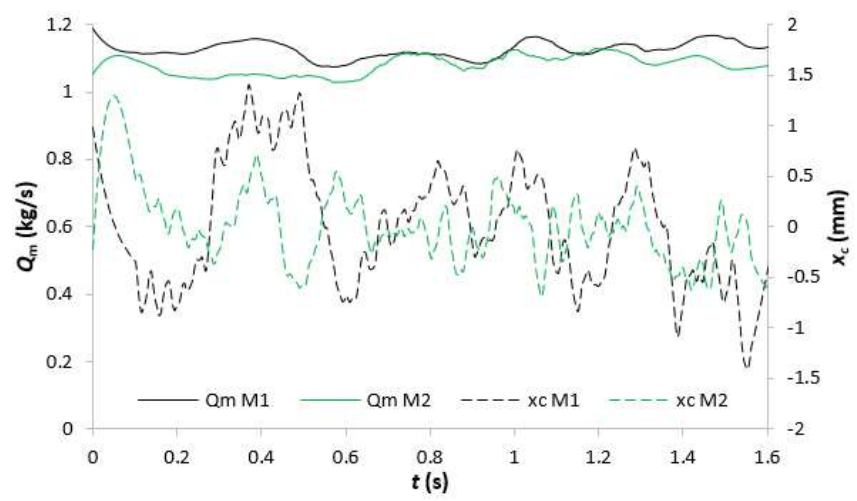

Fig. 16. Measured mass flow rate and centerline position of melt.

Mean melt flow rates for measurements 1 and 2 were $1.12 \mathrm{~kg} / \mathrm{s}$ and $1.08 \mathrm{~kg} / \mathrm{s}$, respectively. This indicates a good agreement with melt production capacity reported by the line operator $(4.0-4.3 \mathrm{t} / \mathrm{h}=$ $1.11 \ldots 1.19 \mathrm{~kg} / \mathrm{s}$ ), and at the same time a good repeatability of measurements, considering the similar $Q_{\mathrm{m}}$ values obtained for both measurements. Moreover, the amplitude of fluctuations of $Q_{\mathrm{m}}$ and $x_{\mathrm{c}}$ is similar for both measurements. While it is not possible to provide exact figures for measurement uncertainty without accurate reference measurements, it is reasonable to estimate that the deviation of $Q_{\mathrm{m}}$ from the true melt flow rate is approximately $5 \%$. 


\section{CONCLUSIONS}

In this paper, a contactless optical method for mass flow measurement of melts has been presented and evaluated. Currently, such optical measurements seem to be the only possibility for real-time flow metering of non-conductive melts (e.g. mineral melt for stone wool production). Experiments in a laboratory set-up show a good agreement between the flow rate obtained by presented optical method and reference measurements by weighing. For stable melt flows with constant or slowly changing flow rate, deviations between optically measured and reference mass flow rates of approximately 5\% can be expected, whereas during rapid flow transitions, the deviation may temporarily rise to about $20 \%$.

With that said, changes in the melt flow rate are typically slow in industrial production lines, meaning that measurement errors will likely be in the range reported for steady-state flows. This is also suggested by results of the second part of this study, where mass flow rate of mineral melt was measured at a stone wool production line. Given the wide range of flow rates successfully measured in this study $(0.05-1.2 \mathrm{~kg} / \mathrm{s})$, and the relatively low cost of imaging hardware required (high definition industrial cameras with 100-120 frames per second), the presented flow measurement method has a good potential for application in stone wool production lines and other industrial processes where precise melt flow metering is required. Nevertheless, we are certain that the accuracy of presented method can be further improved by implementing more advanced edge detection and velocimetry algorithms.

Funding Information. Slovenian Research Agency (ARRS) (Z7-8271), Abelium d.o.o.

Acknowledgment. The authors acknowledge the project Z7-8271 (Improvement of the mineral wool fiberization process) was financially supported by the Slovenian Research Agency and cofinanced by the company Abelium d.o.o. The authors would also like to thank the Department of Metallurgy and Materials at the Faculty of Natural Sciences and Engineering in Ljubljana for their kind provision of experimental facilities.

\section{References}

1. G. Wineland, Conveyor belt scale system: US Patent Nr. US7750253, (2010).

2. B. Širok, B. Blagojević, and P. Bullen, Mineral wool: production and properties (Cambridge: Woodhead, 2008), ISBN 978-1845694067.

3. M. S. Beck and A. Plaskowski, Cross Correlation Flowmeters, Their Design and Application (Bristol: Hilger, 1987), ISBN 978-0852745328.

4. C.W. Fernandes, M. D. Bellar, and M. M. Werneck, "Cross-CorrelationBased Optical Flowmeter," IEEE Transactions on Instrumentation and Measurement 59(4), 840-646 (2010).

5. T. P. Da Silva and F. C. Castaldo, "Ultrasonic anemometer based in cross correlation," in 2015 IEEE 13th Brazilian Power Electronics Conference and 1st Southern Power Electronics Conference (COBEP/SPEC 2015), pp. 1-5.

6. P. Štohanzlová and R. Kolář, "Flow rate estimation by optical coherence tomography using contrast dilution approach," in Optical Coherence Imaging Techniques and Imaging in Scattering Media, B. Bouma and M. Wojtkowski, eds., Proc. SPIE 9541, Optical Coherence Imaging Techniques and Imaging in Scattering Media, 954107 (2015).

7. N. Dubovikova, C. Karcher, and Y. Kolesnikov, "Velocity and flow rate measurement of liquid metal by contactless electromagnetic Lorentz force technique, "IOP Conference Series: Materials Science and Engineering 143(1), 012022 (2016).

8. J. Chen, M. Hočevar, and B. Širok, "Melt Volume Flow Measurement in the Mineral-Wool Production Process," Strojniški vestnik - Journal of Mechanical Engineering 57(4), 293-303 (2011).
9. J. Chen, M. Hočevar, M. Eberlinc, and B. Širok, "Measurement and research on melt mass flow in mineral-wool production process," Yíqi yíbiăo xuébào, ISSN 0254-3087 34(3), 596-601 (2013).

10. "Basler ace acA 1300-30gm," https://www.baslerweb.com/en/products/cameras/area-scancameras/ace/aca1300-30gm.

11. "Bench scale KERN FKB-A," http://www.finemech.com/mm5/4.24/kern_datasheets/sl-fkb-a-gb.pdf.

12. L. D. Pye, A. Montenero, and I. Joseph, Properties of Glass-Forming Melts (Boca Raton: CRC Press, 2005), ISBN 978-1-5744-4662-3.

13. F. Trdič, B. Širok, P. R. Bullen, and D. R. Philpott, “Monitoring mineral wool production using real-time machine vision," Real-Time Imaging 5, 125-140 (1999).

14. B. Bizjan, B. Širok, J. Drnovšek, and I. Pušnik, "Temperature measurement of mineral melt by means of a high speed-camera," Applied Optics 54(32), 7978-7984 (2015).

15. D. I. Gerogiorgis, D. Panias, and I. Paspaliaris, "Multiphysics CFD Modeling of a Free Falling Jet during Melt-Blowing Slag Fiberization," in CFD Modeling and Simulation in Materials Processing, L. Nastac, L. Zhang, B. G. Thomas, A. Sabau, N. El-Kaddah, A. C. Powell, and H. Combeau, eds. (Hoboken: John Wiley \& Sons, 2012), pp. 81-88.

16. J.O. Cruickshank, "A Similarity Between Plane and Axisymmetric ViscousGravity Jets," Journal of Fluids Engineering 106(1), 52-53 (1984).

17. M. Raffel, C. E. Willert, S. T. Wereley, and J. Kompenhans, Particle Image Velocimetry, A Practical Guide (Heidelberg: Springer, 2007), ISBN 978-3540-72308-0.

18. K. Adachi, K. Tagashira, Y. Banba, H. Tatsumi, H. Machida, and N. Yoshioka, "Steady Laminar Round Jets of a Viscous Liquid Falling Vertically in the Atmosphere," AIChE Journal 36(5), 738-745 (1990).

19. U. S. Sauter, H. W. Buggisch, "Stability of initially slow viscous jets driven by gravity," Journal of Fluid Mechanics 533, 237-257 (2005).

20. T. Massalha and R. M. Digilov, "The shape function of a free-falling laminar jet: Making use of Bernoulli's equation," American Journal of Physics 81, 733-737 (2013).

21. "MathWorks Corr2: 2D correlation coefficient," https://www.mathworks.com/help/images/ref/corr2.html. 